\title{
Intelligent Fault Inference of Inverters Based on a Three-Layer Bayesian Network
}

\author{
Sumin Han $\mathbb{D}$, Yongsheng He $\mathbb{D}$, Shuqing Zheng $\mathbb{D}$, and Fuzhong Wang $\mathbb{D}$ \\ School of Electrical Engineering and Automation, Henan Polytechnic University, 454000 Jiaozuo, China \\ Correspondence should be addressed to Sumin Han; hansumin@hpu.edu.cn
}

Received 12 April 2019; Accepted 12 May 2019; Published 16 June 2019

Academic Editor: Denizar Cruz Martins

Copyright (C) 2019 Sumin Han et al. This is an open access article distributed under the Creative Commons Attribution License, which permits unrestricted use, distribution, and reproduction in any medium, provided the original work is properly cited.

\begin{abstract}
A three-layer Bayesian intelligent fault inference model (BIFIM) for an inverter is established to infer the probable uncertain faults. The topological structure of the BIFIM includes the inverter's operation conditions for the first layer, the inverter's faults for the second layer, and the fault symptoms for the third layer, which combines the field technicians' knowledge and experiences with historical running data. The prior probability table of the root node is acquired by the method of basic probabilities corrected historical operation data. The conditional probability parameter table of the BIFIM is obtained by the improved maximum expectation algorithm. Four kinds of incomplete evidence were reasoned and verified, including simple evidence with obvious support, incomplete evidence information, complex evidence without obvious support, and evidence with information conflict. The proposed strategy can make use of the available evidences to inference the probabilities of faults, indicating different reasoning abilities under the different degree of completeness of evidence, especially demonstrating the same inference result under some incomplete evidence information as under complete evidence information.
\end{abstract}

\section{Introduction}

The inverter-powered motor speed control system has now become an industry trend [1] since inverters are widely used in electric drives such as the motor drive and power conversion for its good performance and energy efficiency. Due to the fragility and complexity of power electronic devices, inverters are vulnerable to failure $[2,3]$. During a running process of industrial system, continuity of a specific production process does not allow the system to stop; otherwise it will cause significant economic losses [4]. In recent years, research on fault-tolerant control systems has been given more and more attention $[5,6]$. Fault-tolerant control is often used in inverters to enhance the reliability of the converters, and only detecting accurately and positioning faults can realize fault-tolerant control [7]. The fault inference of inverters is an important means to find and eliminate faults in time and ensure the good operation of the converters, which plays an insightful part in the management, maintenance, and reparation of the machine using inverters. Hence, it is promising to study the fault inference method of inverters. Typically, the failure and symptoms of a device are nonlinear, staggered coupling relationships [8]. A variety of faults may show the same or several symptoms and a failure may also show a variety of more obvious symptoms. Hence, the casual relationship is more complicated and cross-coupled. In addition, due to the limitations of the technical conditions and hardware equipment [9], the collected information of the symptoms is incomplete, is inaccurate, and may even conflict with one another, so the fault inference and diagnosis of complex situations are urgent problems to be solved.

The fault inference method can be divided into three types: the artificial intelligence (AI), the fault tree, and a contrast detection. Firstly, AI method generally includes artificial neural networks [10], genetic algorithms (GA) and program design $[11,12]$, the expert system $[13,14]$, the fuzzy rough set theory [15], and other methods. In [10], a neural network was applied to the fault reasoning of a rotating machinery, which employed multisensors' information to implement deep learning. Wang et al. [11] proposed an improved GA to develop power transforms' faults inference, which found the best parameters of the classical domains and weights in the extended clustering method through EGA's search characteristics. Mohammed, M. A. et al. [12] proposed 
a hybrid scheme that combines the GA with case-based reasoning to improve CBR diagnosis effect. An instinctive transformer's fuzzy expert system for multifault diagnosis was presented [13]. In [14], through the accumulation of experience, the study listed the possible failures, summed up the principles, and built an expert system based on a knowledge base to determine the fault type of internal combustion engines. Saravanan et al. [15] used a data mining system to form a decision-making system by distinguishing the features of various fault conditions using a decision tree. Secondly, the fault tree mode diagnosis method includes the traditional fault tree methodology [16] and fuzzy failure tree means [17]. Ni, S. X. et al. in [16] built a fault tree, which was established by using the minimum cut set method to determine the breakdown in the case of power system. Gharahasanlou, A. N. et al. in [17] applied the fault tree to the fault inference for the crushing and mixing bed hall. Finally, the contrast detection method includes the current detection one [18] and voltage detection one [19]. The authors in [18] analyzed the current characteristics of a motor under general states and open circuit fault states, employing the sum value of the modulus of the three phase currents as the characteristic quantity for fault inference. In [19], fault diagnosis was carried out by comparing the difference between the voltages of the inverters in normal state and in the faulted states.

The above methods can be used for fault inference and the diagnosis of inverters, but most of them need complete and complete fault characteristic information and fault data. However, it is difficult to collect accurate and complete information about the symptoms in practice; the data obtained in most cases are incomplete and even the non-target data is conflicted. Therefore, in the case of incomplete information, it is crucial to research the relatively intelligent fault inference method with domain expert knowledge and to make inference and diagnosis.

To consider the environmental conditions and working conditions, researchers in different fields have established specific three-layer Bayesian Network (BN) containing such factors as a layer [8, 20-22]. Bin Gang Xu [8] constructed a three-layer BN including machine operating state layer to diagnose four types of faults of a flexible rotor: rotor unbalance, rotor crack, etc. Zengkai Liu [20] et al. proposed a threelayer $\mathrm{BN}$ based on empirical mode decomposition method to diagnose the faults of gear pumps. In addition to the usual fault layer and fault symptom layer, the $\mathrm{BN}$ also contains a multisource information layer, which considers the factors such as human observation information, system maintenance information, or abnormal operation records. Literatures [21, 22] proposed three-layer BNs containing environmental information or operational status for internal combustion engines and power quality analysis. The validation results showed that three-layer BNs can better combine historical experiences and equipment information for more intelligent fault diagnosis and accurate reasoning. However, the application of three-layer $\mathrm{BNs}$ to the fault diagnosis of power electronic equipment has been not deeply studied by far.

In the study, the intelligent fault inference model for an inverter employing the Bayesian network (BN) is developed

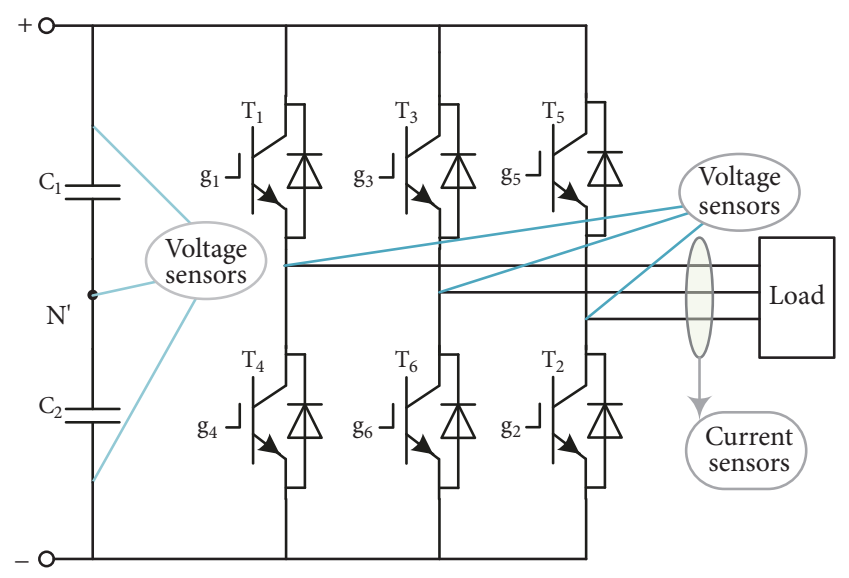

FIGURE 1: Topology structure of a typical three-phase bridge inverter.

with the knowledge of domain experts, inverter operating states, inverter faults, and fault symptoms. Based on relevant knowledge and experiences, a 3-layer Bayesian belief structure is established and the corresponding probabilities distribution table (CPT) of all the nodes is determined. Compared with the existing Bayesian network for reasoning, the major contributions of the study are as follows.

(1) A three-layer Bayesian intelligent fault inference model (BIFIM) is proposed, in which the operating state layer of equipment is added to reflect the operating state and working conditions of equipment. The basic probability correction method for historical operation data of root nodes and the improved maximum expected conditional probability method for nonroot node are proposed, respectively.

(2) Based on the proposed three-layer Bayesian intelligent fault inference model, probabilistic explanations can be obtained for faults of various types, especially for incomplete evidence information demonstrating the same inference result under some incomplete evidence information as under complete evidence information. The proposed model can intelligently infer the fault probability results under a variety of complex situations by synthesizing the operating data and evidence information of the equipment, which shows the intelligence of the reasoning model.

The paper is organized as follows: Section 2 describes the system principle of a typical and widely used inverter and illustrates the fault features under open circuit states. Section 3 discusses the Bayesian intelligent fault reasoning algorithm framework, including message passing principle. The establishment of the three-layer BIFIM including the architecture and the probability parameters is demonstrated in Section 4. The integrated inference and deep analysis of the proposed approach under various evidence information and operating states are provided in Section 5. Finally, the conclusions about the proposed three-layer BIFIM for the invert are presented in Section 6.

\section{System Description and Fault Features}

The topology structure of a typical three-phase voltage source inverter driving mine hoisting is illustrated in Figure 1, 

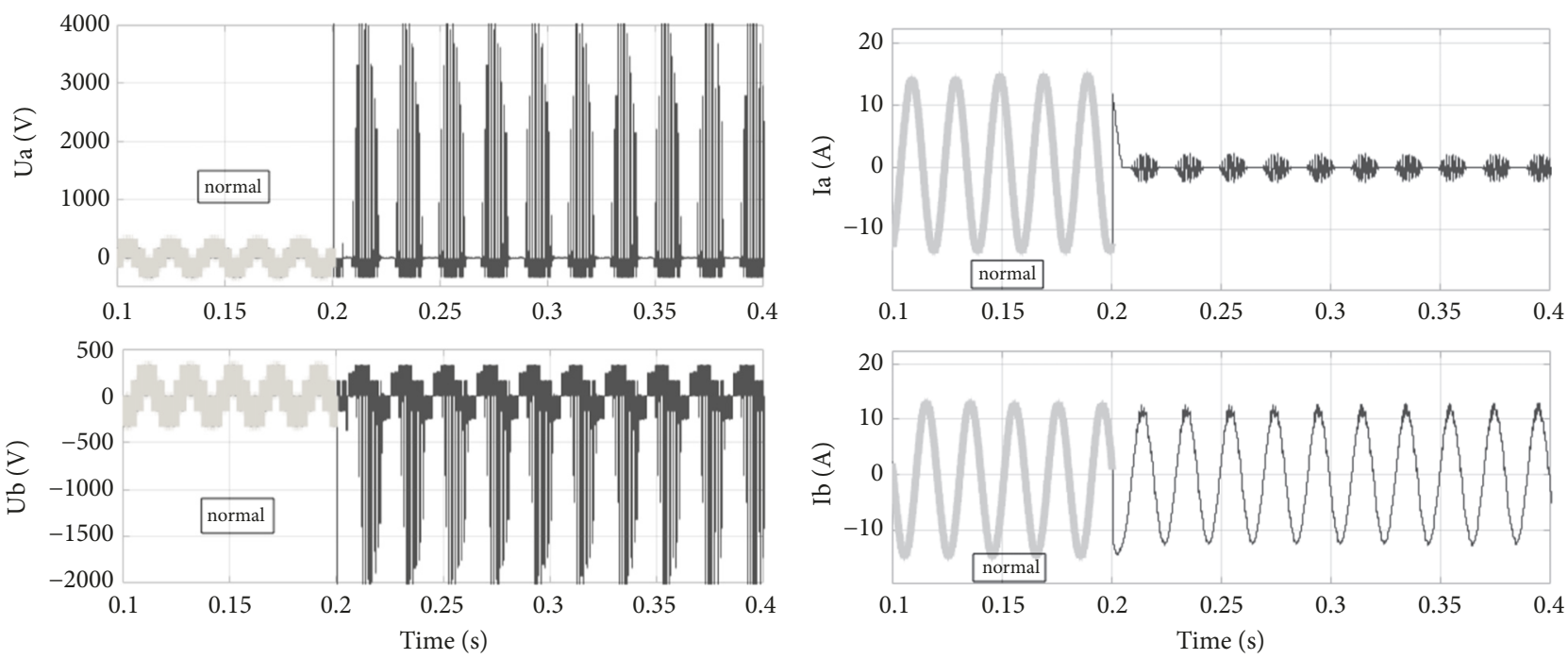

(a)

(b)

FIgURE 2: Experimental phase voltages Ua, Ub, currents Ia, Ib with OC fault occurred in $\mathrm{T}_{1}$.
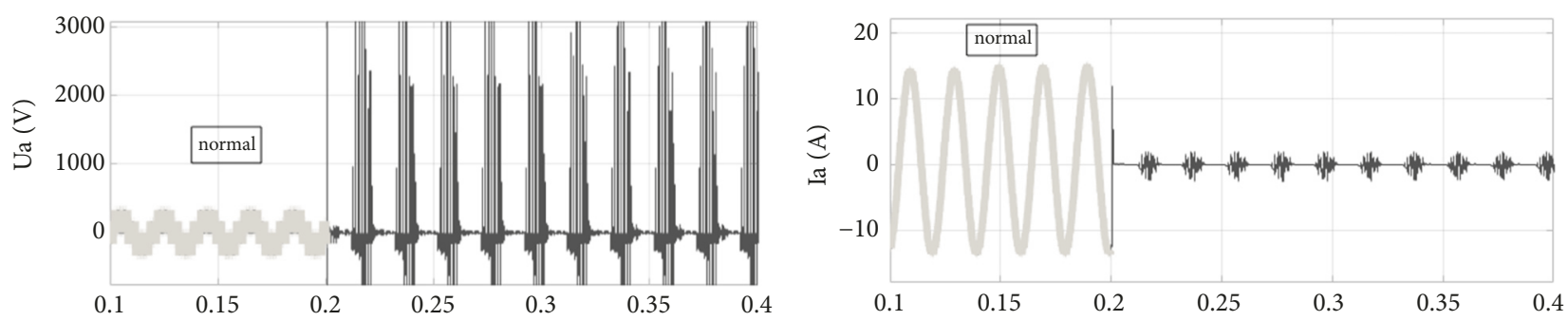

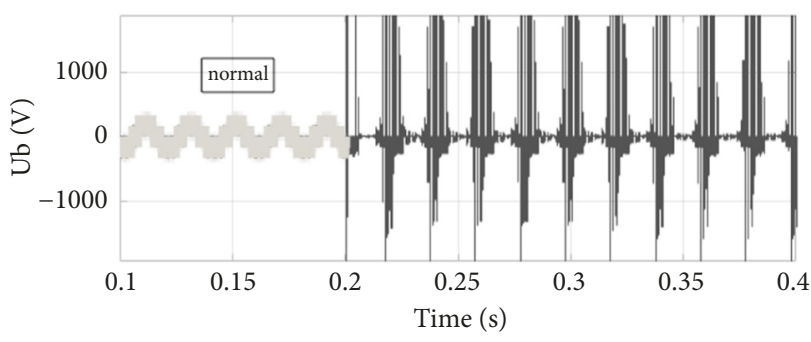

(a)

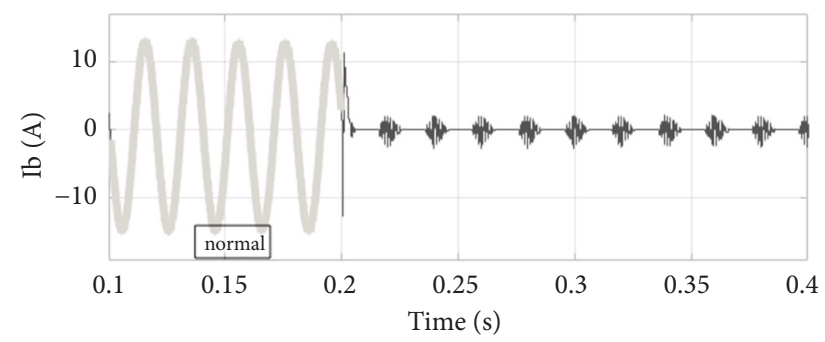

(b)

FIGURE 3: Experimental phase voltages Ua, Ub and currents $\mathrm{Ia}$, Ib with OC fault occurred in $\mathrm{T}_{1}, \mathrm{~T}_{3}$.

including a DC link and a three-phase bridge module. The DC link is composed of two large equivalent capacitors to maintain the DC voltage smooth and buffer passive energy. The three-phase bridge module is called the main circuit, composed of six bridge arms. Each arm has a power switch $\mathrm{T}_{i}$ (IGBT, $\left.i=1, \ldots, 6\right)$ and a counter parallel diode. The conduction mode of each phase is $120^{\circ}$ and that of a pair of bridge arm up and down is $180^{\circ}$. Three-phase sinusoidal voltages of different frequencies can be obtained by using different PWM control modes, by which a series of modulated pules can be generated to exert on the terminals $\mathrm{g}_{i}(i=$ $1, \ldots, 6)$. Consequently, the voltage sensors acquire the phase voltage waveforms of the three phases in the normal state, shown in Figure 2(a). Similarly, the current sensors acquire the current waveforms of the three phases in the state, shown in Figure 2(b).
The power switches and the DC link of the inverter circuit are high frequently happening fault positions shown in Figure 1. In practice, 1 IGBT appearing open circuit (OC) fault accounts for the majority, and there are 6 modes $\left(T_{i}\right.$, $i=1, \ldots, 6)$ in total. In the high-power converter circuit, there are often 22 kinds of faults simultaneously happening in the two switches, which cause serious damages. However, three switches' failure at the same time is less likely, which involves 20 modes totally. As can be seen from Figures 2(a) and 2 (b), when $t \geq 0.2 \mathrm{~s}$, an OC fault occurs in a switch (e.g., $\mathrm{T}_{1}$ ). The phase currents at the output side of the same phase in the inverter will be seriously distorted, while the voltages of phases A, B, and C will all be distorted, resulting in highvoltage pulses and high-order harmonics. If two switches of different phases have OC faults at the same time (e.g., $\mathrm{T}_{1} \mathrm{~T}_{3}$, Figure 3 ), the currents of the both phases will be seriously 
distorted, and the voltages of $\mathrm{A}, \mathrm{B}$, and $\mathrm{C}$ phases will be distorted. If two switches of the same phase are open at the same time, only the voltage and current of that phase are distorted, while currents and voltages of the other phase will not be influenced. In addition, when the OC fault is caused by invalidity of the drive signal, a sparse pulse strings will appear in the voltage waveform at the corresponding output side.

From Figures 2 and 3, when the OC fault occurs in one or more power switches of the inverter, the three-phase voltages and currents will be distorted, demonstrating obvious differences with different fault modes. Therefore, it is feasible to collect the voltage and current waveforms to extract the fault features.

\section{Bayesian Diagnostic Inference Algorithm Framework}

A Bayesian diagnostic inference is an inference form that can find out the causes of the result and calculate the probabilities of its occurrence when the result is known. The result referred to the symptom information which can be complete or incomplete and the cause is likely to one or more. Namely, the inference result of the Bayesian diagnostic network describes the probability order of all the causes, which determines the occurring fault under certain inference rules. It is usually used in the case of fault diagnosis $[23,24]$.

3.1. Bayesian Diagnostic Inference Process. The work flow of the Bayesian diagnostic inference network is shown in Figure 4. Firstly, according to the field technicians' knowledge and experiences or structure learning algorithms, the Bayesian network structure can be established. By the acquired data samples, we can calculate the prior probabilities by parameter algorithms or empirical methods for root nodes, while for leaf nodes parameter algorithms are suitable to obtain conditional probabilities due to the huge data. Secondly, we obtain the evidence information by the collected symptom information, updating the messages of each node through message passing. Finally, the study calculates the probability distribution of the query node, which needs to calculate its probability of occurrence or not.

The most fundamental step in the diagnostic inference procedure is the construction of a target Bayesian network and the acquisition of the prior probability of each node, and the most critical step is message passing. The message passing algorithm is suitable for single connected networks, and its computation is simple and fast. In Section 4, the establishment process of the diagnostic inference Bayesian network of inverters is given. In this section, the principle of message transmission is described in detail.

3.2. Message Passing Principle. The Bayesian network displayed in Figure 5 contains two root nodes $(A, B)$, three intermediate nodes $(C, D, E)$, and four-leaf nodes $(F, G$, $H, I)$. The message passing structure is composed of nodes of variables in the Bayesian network. The ellipse stores the newest probability information. The message from a parent knot to its child is named a $\pi$ message, and the message from a child knot to its parent node is called the $\lambda$ message $[25,26]$.

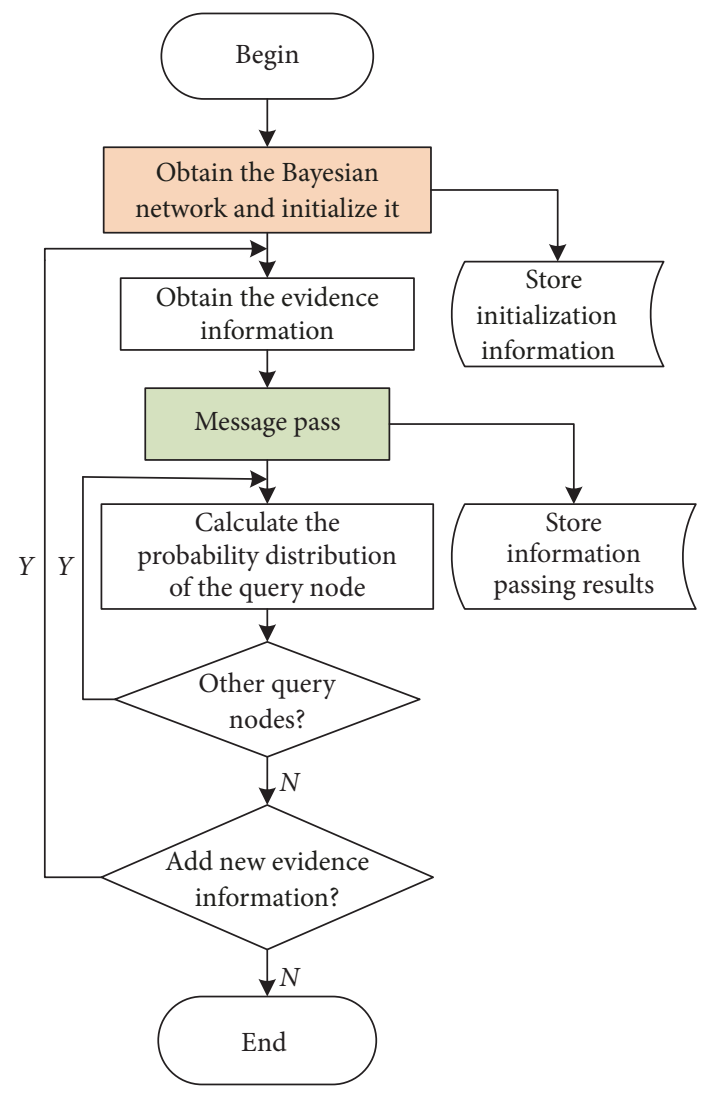

FIGURE 4: The workflow of Bayesian diagnostic inference networks.

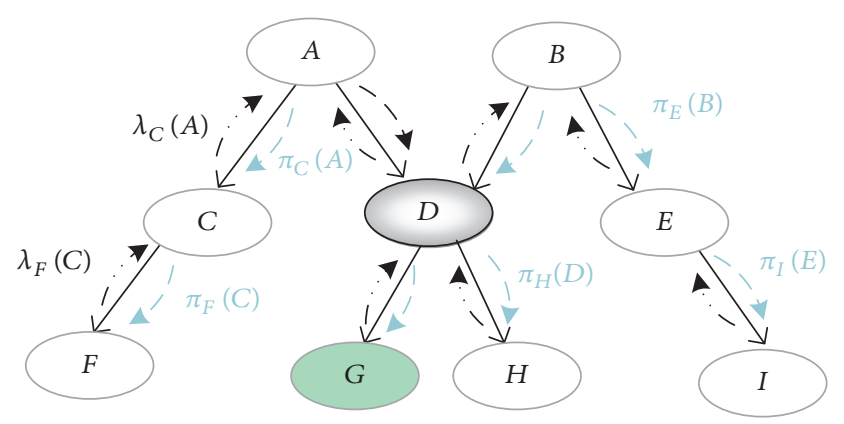

FIGURE 5: Message transmission structure in a Bayesian network.

Some $\pi$ messages and $\lambda$ messages are given in Figure 5; e.g., $\lambda_{F}(C)$ denotes the $\lambda$ message from the child knot $F$ to its parent knot $C$.

Message passing course is broken into two phases: message collection stage and the message distribution stage [25]. The two stages collect and distribute messages through a hub node and then obtain the probability of each parent node according to the evidence information. We give an example to analyze and demonstrate the massage passing principle $[26,27]$. For example, the proof information $G=g$ is given in Figure 5 and $D$ is selected as the hub node to analyze the message passing algorithm principle. The algorithm requires the initializations for all the $\pi$ and $\lambda$ messages: set all the 
values of $\pi$ and $\lambda$ messages to land $\pi$ messages of root messages to the prior probabilities.

3.2.1. Message Propagation Algorithm. (1) For any node $S$ ( $S$ $=F, C, A, D, H, I, E, B)$ that is not an evidence node, $S$ receives all messages from its subnodes to obtain $\lambda\left(s_{i}\right)$, which is calculated through

$$
\lambda\left(s_{i}\right)=\prod_{q} \lambda_{Y_{q}}\left(s_{i}\right)
$$

where $Y_{q}, q \in[1, m]$ is the child node of the node $S . s_{i}$ means the $i$ th state of node $S$. If the parent node has only one child node, $\lambda\left(s_{i}\right)$ is just the message itself. If there are multiple child nodes, $\lambda\left(s_{i}\right)$ is the product of several messages. For any node $\mathrm{S}$, the $\lambda$ message corresponding to any state of its parent node is

$$
\lambda_{S}\left(u_{i}\right)=\sum_{s_{i}} \lambda\left(s_{i}\right) \sum_{u_{k}: k \neq i} P\left(s_{i} \mid u_{1}, \ldots, u_{m}\right) \prod_{k \neq i} \pi_{S}\left(u_{k}\right)
$$

where $u_{i}, i \in[1, n]$ is the $i$ th state of node $S$ 's parent node $U$.

If node $S$ is the evidence node and the observed state is $s_{e}$, the received message is shown in

$$
\lambda\left(s_{i}\right)= \begin{cases}0 & i \neq e \\ 1 & i=e\end{cases}
$$

(2) Any node $S$ (except evidence nodes) receives all messages from its parent node to obtain $\pi_{s_{i}}\left(u_{j}\right)$. New $\pi$ messages of node $S$ are calculated through

$$
\begin{aligned}
\pi_{Y_{j}} & \left(s_{i}\right) \\
& =\prod_{k \neq j} \lambda_{Y_{k}}\left(s_{i}\right) \sum_{u_{1}, \ldots, u_{m}} P\left(s_{i} \mid u_{1}, \ldots, u_{m}\right) \prod_{i} \pi_{S}\left(u_{i}\right)
\end{aligned}
$$

If node $S$ is the evidence node and the observed state is $s_{e}$, the received message is shown in

$$
\pi_{Y_{j}}\left(s_{i}\right)= \begin{cases}0 & i \neq e \\ 1 & i=e\end{cases}
$$

(3) Belief updating is as follows.

When a node $S$ receives the $\pi$ messages from the parents and the $\lambda$ messages from the children, it indicates that the belief parameter of the node has been updated by the following equation.

$$
P\left(s_{i} \mid e\right)=\sigma \lambda\left(s_{i}\right) \pi\left(s_{i}\right)
$$

where $e$ is the evidence given and $\sigma$ is the normalized function rendering $\sum_{i} P\left(s_{i} \mid e\right)=1$.

3.2.2. Message Passing Process. The two stages collect and distribute messages through a hub node, through which evidences and information passes as much as possible according to the evidence nodes and the target nodes. Then, $D$ is selected as a hub node when $G$ is an evidence node. The message passing process is as follows.

(1) Parameters Initialization. In the stage before any evidence is entered, all the $\pi$ messages of root nods $\pi(A)$ and $\pi(B)$ are initialized from the priors. All the $\lambda$ messages of leaf nodes without evidence set $\lambda(F)=(1,1), \lambda(G)=\lambda(H)=\lambda(I)=$ $(1,1)$.

(2) Propagation without Evidence. In the stage, node $D$ updates the belief with (6) with no evidence, $P(A)$ and $P(B)$. $\pi_{D}(A)$ and $\pi_{D}(B)$ are calculated with (4). According to (2), $\lambda$ messages will not change with no evidence. Consequently, node $D$ can compute its own $\pi$ message and pass down to its children $G$ and $H$. Meanwhile, the other nodes $C, E$, etc. also receive messages from their parents and sent out messages to their children. Since these nodes have no effect on the diagnosis result with no new message updating, there is no necessity to discuss the corresponding message propagation.

(3) Propagation with Evidence. When evidence $G=g$ is entered by setting $\lambda(G)=(1,0)$, the message $\lambda_{G}(D)$ will be calculated and sent up to $D . \lambda(D)$ and $P(D \mid$ e) are then reupdated. Accordingly, the new messages are sent to $D$ 's parents and to its other children. Therefore, the belief of root nodes $P(A \mid \mathrm{e})$ and $P(B \mid \mathrm{e})$ is recomputed.

Hence, the Bayesian diagnosis inference network can make a probabilistic interpretation for a complex system with multiple causes and multiple faults and can provide more objective data judgment under incomplete information.

3.3. The Intelligent Three-Layer Bayesian Network Frame. In the field of failure diagnosis and reasoning, the operation states and operating conditions for equipment have a great influence on the reasoning results. If the working parameters of a certain piece of equipment are known, many other faults can be excluded even if the fault evidence is incomplete, and the probability of a certain fault may increase rapidly. Thus, a three-layer Bayesian fault inference network based on the operational state layer is proposed in this paper as shown in Figure 6. In Figure 6, the structure from top to bottom is constituted of the running condition layer, fault layer, and fault omen layer. Accordingly, domain experts and fault data are required to obtain the 1st layer's prior probabilities and conditional probabilities of the 2 nd and 3rd layers. When the inference network's parameters are obtained, the fault probability inference can be carried out based on the evidence of the third layer, and, of course, two-way inference can also be implemented in combination with the operational state layer.

As shown in Figure 6, in the Bayesian network frame diagram, the first layer is the operation condition layer, which mainly reflects the inverters' running states and bad working conditions. Generally, the variables listed in this layer are all kinds of faults that lead directly to the converter such as pulse loss of a phase, capacitance damaging, or aging. The variables in this layer are the root nodes in the Bayesian network, and the prior probabilities are evaluated by the specialists and then improved through the technicians as the inverter's 


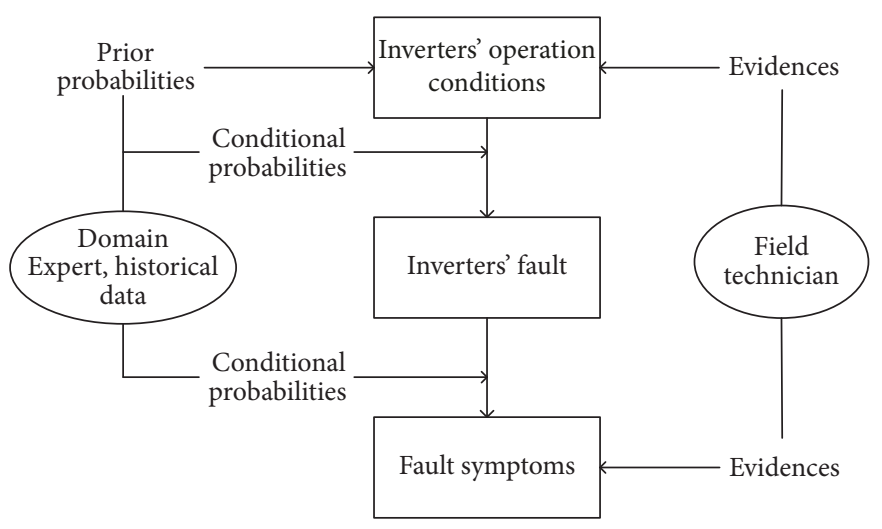

FIGURE 6: A proposed three-layer Bayesian network framework for inverters.

operating circumstances are more complex due to the field work environment, emergency, load fluctuations, operating errors, and the influence of other factors [28, 29]. Hence, the variables of this layer should be determined by domain experts and field technicians based on the field operation status and historical data.

The variables listed in the second layer are various faults of inverters or the positions of faults, and the common faults of inverters are as follows: A-phase failure, B-phase fault, C-phase fault, and DC-link fault. The variables listed in the third layer are the diverse symptoms of inverters such as waveform distortion, abnormal pulse, abnormal voltage, and waveform distortion. The faults in the second layer are mainly reflected by the fault symptoms of the third layer after feature extraction from some monitoring data. The structure of the 1st and 2nd layer and their causal relationships are determined by domain experts and field technicians. Finally, the evidence needed for inference comes mainly from the third-layer fault symptoms as well as the first-layer inverters' operating conditions, both of which are provided by symptom data and historical running data.

In contrast with the traditional Bayesian network structure (which contains only two layers: inverters' fault layer and symptom layer [30]), the three-layer BN structure proposed in this study considers both the inverters' faults and fault omens and the operational circumstances that may lead to the inverters' failure. In the actual project, these results in the operation of the inverter's fault can provide a lot of useful diagnostic information and can enhance the precision of fault inference.

\section{Establishment of the Fault Inference Network for Three-Layer Bayesian Inverters}

In the section, an improved $\mathrm{BN}$ structure for fault inference applied to inverters named BIFIM is structured, which has a three-layer structure: the operation conditions layer, the inverters' fault layer, and the fault omens layer. Thus, the construction consisted of two causal topology structures that involves more information about the operating conditions of the equipment and the symptoms of the failure. The three layers have a direct causal relationship in turn.
The specific work flow of the Bayesian network is shown in Figure 4. In view of the characteristics of the OC faults of the three-phase voltage source inverter (Section 1), the fault's causality is analyzed and the structure of the BIFIM is established. Parameter learning methods from the acquired fault symptom data or else can obtain the prior probabilities and conditional likeliness of the improved BN. Uncertain and incomplete inference shall be made according to the evidences.

4.1. Topology of Bayesian Fault Inference Network for the Inverter. From the above analysis, the second layer of BIFIM consists of four nodes: A-phase failure, B-phase fault, C-phase fault, and DC-link fault. Each phase failure may be caused by the upper switch fault and the lower switch fault, and the fault of each bridge arm may be the OC fault of this switch or the fault of this drive pulse. Therefore, of all the A, B, and $C$ phase faults each fault has four parent nodes. The fault of DC link may be caused by two capacitor's faults or capacitor parameter aging and weakening, which has four parent nodes accordingly. The symptoms of each phase fault can be shown as positive and negative half-cycle distortions of waveforms of each phase and abnormal pulses of voltage waveforms. Therefore, of all the A, B, and C phase faults each fault has three child nodes, while the possible symptoms of DC link fault are both the positive and negative half cycle waveform distortion of voltages and currents in all phases, in addition to two capacitor voltages' distortion.

Accordingly, the topology of the BIFIM of the inverter is shown in Figure 7, which consists of 29 variable nodes. Among them, there are 15 nodes $X_{1}-X_{15}$ for the inverter's operation conditions layer, four nodes $X_{16}-X_{19}$ for the inverters' fault layer (shown in the highlighted ovals), and 10 nodes $X_{20}-X_{29}$ for the fault omens layer. Moreover, the prior likeliness and conditional likeliness required of Bayesian networks are obtained through statistical data and practical experience.

4.2. Data Preprocessing. Each node in the BIFIM has two working states. For the variables in the first and second layers, they are state variables with two values, normal or failure. As for the variables in the third layer, they are the data 


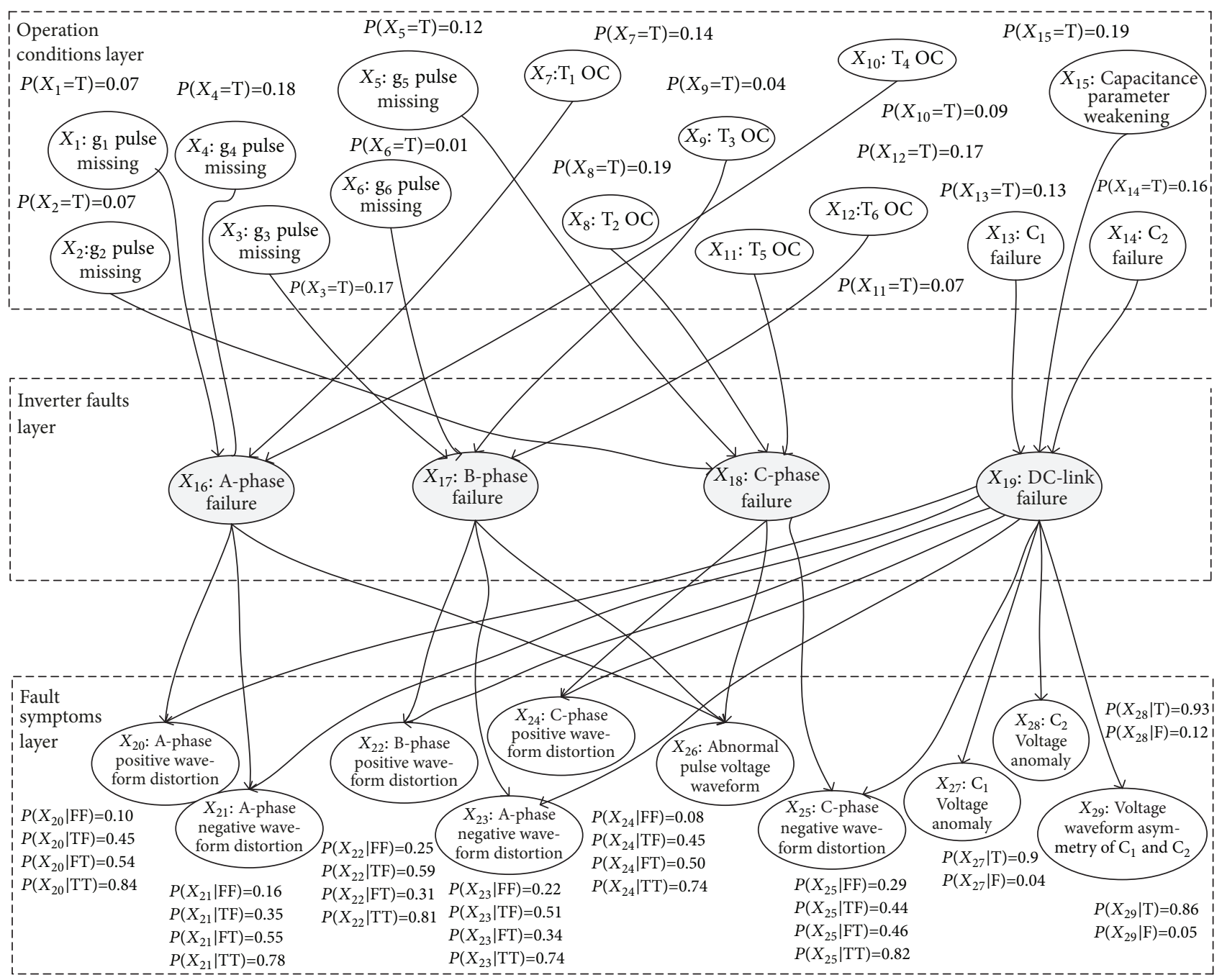

FIgURE 7: The BIFIM of the inverters' fault inference.

variables which require data preprocessing, especially for the waveform distortion rate and abnormal pulse of waveforms. As mentioned above, A, B, and C phase voltages, currents, and capacitor voltages are taken as the detection variables. Then the quantization formula of positive and negative half-cycle waveform distortion data of voltage and current is

$$
\begin{aligned}
& \frac{\sum_{j}\left(x_{j}-x_{j i}\right)}{\sum_{j} x_{f i}}= \begin{cases}<-5 \% & \mathrm{Q}_{i}=1 \\
\geq-5 \% & \mathrm{Q}_{i}=2\end{cases} \\
& \frac{\sum_{j}\left(x_{j}-x_{j i}\right)}{\sum_{j} x_{f i}}= \begin{cases}>5 \% & \mathrm{~W}_{i}=1 \\
\leq 5 \% & \mathrm{~W}_{i}=2\end{cases}
\end{aligned}
$$

where $\mathrm{Q}_{i}$ represents the variable corresponding to $X_{21}, X_{23}$, and $X_{25}$ in layer 3; i.e., $i=21,23,25 ; W_{i}$ represents the variable corresponding to layer $X_{20}, X_{22}$, and $X_{24}, i=$ $20,22,24 ; x_{j}$ is the $j$ th extracted value for variable $X\left(\mathrm{Q}_{i}\right)$ in a given period, and $x_{f j}$ is the $j$ th extracted value for variable $X\left(\mathrm{Q}_{i}\right)$ in a given period when it runs normally and stably.
Similarly, for $X_{27}$ and $X_{28}$, we employ the absolute value of (7)-(8) to realize the quantization.

For the case that the abnormal waveform of voltage and current occurs in all the three phases, the abnormal pulse voltage signal reflecting the abnormal pulse in the three phases simultaneously can be used as the statistical quantification equation:

$$
\begin{gathered}
T H_{A} \& T H_{B} \& T H_{C}= \begin{cases}1 & \mathrm{~V}_{i}=1 \\
0 & \mathrm{~V}_{i}=2\end{cases} \\
\frac{\left(\sum_{j=2}^{l} \aleph_{j}^{2}\right)^{1 / 2}}{\aleph_{1}}=\left\{\begin{array}{ll}
>3 \% & T H_{i}=1 \\
\leq 3 \% & T H_{i}=0
\end{array},\right.
\end{gathered}
$$

$$
i=A, B, C
$$

where $\mathrm{V}_{i}$ represents the variable corresponding to $X_{26}$ in layer 3; i.e., $i=26$; $\aleph_{i}, i \in[1, l]$ denotes the root mean square value of each harmonic to the variable $X\left(\mathrm{~V}_{i}\right) ; l$ is the maximum harmonic order intercepted. 
TABLE 1: Prior probability correction table of root nodes.

\begin{tabular}{lccccc}
\hline Number & Influencing factors & Factor probability $P_{i}$ & Probability coefficient $a_{i}$ & Correction result \\
\hline 1 & Maintain regularly & $P_{1}$ & $a_{1}=0$ & Yes & No \\
& & & $a_{1}=1$ & & $+a_{1} P_{1}$ \\
2 & Operating years $t_{1}$ & $P_{2}$ & & $a_{2}=t_{1}$ & $+a_{2} P_{2}$ \\
3 & Failure times $t_{2}$ & $P_{3}$ & & $a_{3}=t_{2}$ & $+a_{3} P_{3}$ \\
4 & $\ldots$ & $\ldots$ & $\ldots$ & $\ldots$ \\
\hline
\end{tabular}

4.3. Parameter Probability Table Acquisition. The probability parameters needed in the BIFIM of the inverter are acquired by historical data and practical experience. The parameter probability table is a very important part of the Bayesian network, correct acquisition of which is the basic guarantee of accurate and reliable reasoning. According to the location of nodes, the acquisition methods of probability table can be divided into the prior probability acquisition method of root nodes and acquisition method for conditional probabilities of nonroot nodes. The basic probability correction method for historical operation data of root nodes and the improved maximum expected conditional probability method for nonroot node are proposed and analyzed in the study, respectively.

4.3.1. Basic Probability Correction Method for Historical Operation Data. In the actual operation of the inverter, the operation states and historical data of the equipment have a great impact on the fault location of the inverter. Experienced technicians can basically accurately judge or predict the fault location according to the maintenance records and the field situation. For this reason, the paper proposes the basic probability correction method for historical operation data. For the 15 variables in the first layer of the network as shown in Figure 7, the basic probability is preset first, and then the prior probability is modified according to their service life and whether they are regularly repaired and whether they have failures before.

The formula of the basic probability correction method for historical operation data is as follows:

$$
P=P_{0}+\sum_{i=1}^{c} a_{i} P_{i}
$$

where $c$ is the number of factors influencing the prior probability parameter of a root node; $P_{i}$ is each factor's probability; $P_{0}$ is the basic probability; and $a_{i}$ is the coefficient of each factor. The details are displayed in Table 1 . Table 1 lists three factors that affect the prior probabilities, periodic maintenance, service life, and failure times. If the equipment is maintained regularly, then $a_{1}=0$; otherwise, $a_{1}=1$; i.e., the prior probability of failure of the equipment will decrease under regular maintenance conditions. For the service life, if the service life is long, the probability of failure of the equipment will increase, and the factor is proportional to the service life. For the number of failures, the historical failures and the number of failures will increase the probability of the equipment's failure, which is proportional to the number of failures.
All the root nodes of the inverter are assigned by the above method and (11), and the root nodes' prior probability table is confirmed. The study uses the maintenance records, patrol logs, and test data of the main shaft drive system hoist inverter (Model: ACS6000) in Jiaozuo, Henan Province to calculate the probability of each node. The results of the prior probabilities of the root nodes $\left(X_{1}-X_{15}\right)$ are illustrated in Figure 7.

4.3.2. Improved Maximum Expected Conditional Probability Method. The improved maximum expected conditional probability method is to determine the maximum value of the likelihood function under the condition of missing data [26]. Assume that $N=(G, \Theta)$ is the Bayesian diagnostic inference network with known topology $\mathrm{G}$ and unknown parameters $\Theta, X_{i}$ is the node of the network with $i \in[1, n]$, and $n$ is the number of the nodes in the network. $D=\left(D_{1}, D_{2}, \ldots, D_{\mathrm{m}}\right)$ is a grope of dataset, where $m$ samples are independent mutually. Among the dataset, any sample $D_{r}$ consists of the missing variable $Z$ and the complete observable variable $Y$. In this way, the logarithmic likelihood function containing $m$ probability distribution model parameters is expressed as follows.

$$
L(\theta)=\sum_{i=1}^{m} \log \sum_{n} P\left(Z=z_{i}, Y=D\right)
$$

As $Z$ contains missing information, the maximum value of (12) cannot be directly obtained. Therefore, the probability distribution function $f\left(Z=z_{i} \mid Y\right)$ of the variable $Z$ is introduced to represent the probability $Z=z_{i}$ under the condition of $Y$. Equation (12) can be denoted as

$$
L(\theta)=\sum_{i=1}^{m} \log \sum_{n} f\left(X=x_{i} \mid Y\right) \frac{P\left(X=x_{i}, Y=D\right)}{f\left(X=x_{i} \mid Y\right)}
$$

By adjusting $f\left(X=x_{i} \mid Y\right)$ to make (13) obtain the maximum value, the conditional probability parameter of the Bayesian diagnostic inference network $G$ can be acquired.

$$
\begin{aligned}
\widehat{\theta} & \arg \max _{\theta}\left(\sum_{i=1}^{m} \log \sum_{n} f(X\right. \\
& \left.\left.=x_{i} \mid Y\right) \frac{P\left(X=x_{i}, Y=D\right)}{f\left(X=x_{i} \mid Y\right)}\right)
\end{aligned}
$$

In view of the above analysis, to gain the CPT of the leaf nodes in the BIFIM as shown in Figure 7, the simulation 
TABLE 2: Main parameters of the three-phase voltage inverter.

\begin{tabular}{lc}
\hline Name & Value \\
\hline DC voltage & $500 \mathrm{~V}$ \\
IGBT resistance & $0.001 \Omega$ \\
Nominal phase-to-phase voltage of the load & $220 \mathrm{~V}$ \\
Active power of the load & $2.2 \mathrm{~kW}$ \\
Positive power of the load & $330 \mathrm{Var}$ \\
Modulation frequency $\left(f_{\mathrm{M}}\right)$ & $25-75 \mathrm{~Hz}$ \\
Sampling frequency & $f_{\mathrm{M}} * 32$ \\
\hline
\end{tabular}

model was built in the Matlab/Simulink environment, and the simulation experiment was designed. The main parameters of the simulation experiment are shown in Table 2.

The data to be collected are phase voltages, phase currents of the output side, and two voltages of the DC link, a total of 8 types of signals. The PWM modulation frequency $f_{\mathrm{M}}$ of the IGBT driver circuit is adjusted between $25 \mathrm{~Hz}$ and $75 \mathrm{~Hz}$, changing in increments of $2 \mathrm{~Hz}$. There are 101 groups of data for each signal at each frequency, and 128 sampling points are taken at 4 cycles for each signal. Namely, the sampling frequency of the signal is $f_{M} * 32$, and the sampling frequency shall be set according to different frequencies in the simulation. In each mode, the dimension of each signal to be collected is $8 * 101 * 128$. There are 35 fault modes, including 1 mode under the normal circumstance, 6 modes under single OC (only one switch) fault, 6 modes under single pulse loss and 22 modes under double OC fault (two switches simultaneously). The preliminary data volume obtained is $35 * 8 * 101 * 128$. According to the data quantization formulas described in Section 3.2, the final data volume obtained is $35 *$ $101 * 10$. According to practical experience, the occurrence of a fault is an abnormal event of a small likeliness, and the probability of double OC faults is lower than that of single OC faults. Therefore, the data are randomly selected according to the ratio of 98:15:3 for normal data, single OC fault data and double OC fault data, which are used to do parameter learning according to the method described in Section 4.3. The remaining data samples are taken as test data to verify the accuracy and reliability of the BIFIM. The results of parameter learning are shown in Tables 3 and 4 and Figure 7.

On the second layer of BIFIM, each node has major parent nodes. $X_{16}, X_{17}$ and $X_{18}$ have four parent nodes, while $X_{19}$ has three parent nodes. In addition, given the limited space in Figure 7, the conditional probabilities of $X_{19}$ and $X_{26}$ are listed in Table 3, and the conditional probabilities of X16, $\mathrm{X} 17$ and X18 are listed in Table 4. For example, the probability that $\mathrm{X}_{19}$ occurs when the three parent nodes $X_{13}$ do not occur, $X_{14}$ occurs, and $X_{15}$ is 0.72 .

\section{Integrated Inference of the Inverter Fault}

5.1. Classification of Faults and Evidence. According to the constructed BIFIM, the evidence and faults of the inverter shall be classified and synthesized to infer faults more accurately. First, evidence can be classified according to the following three perspectives.
(1) The Complexity of the Evidence. It can be classified into simple evidence and complex evidence from the complexity of the evidence. Simple evidence is referred to that clearly supports the occurrence of a fault, while complex evidence is defined by that does not obviously support the occurrence of a fault.

(2) Multiple Sources of Evidence. It can be classified into single source evidence and multiple sources evidence from the source of the evidence. If the evidence is from a single layer, it is called single source evidence. If the evidence is from two layers or more, it is called multiple sources evidence.

(3) Completeness of the Evidence. Based on the degree of completion of evidence, it can be divided into complete evidence and incomplete evidence. Complete evidence means that all the evidence at the symptom layer is given and there is no missing data for each symptom node. If any symptom node data cannot be obtained or lost, or some data points are missing for a certain symptom data, it is incomplete evidence.

Although power switches' faults in the main circuit of the inverter occur mostly in a single switch, yet it is inevitable that multiple switches' fault probably occur at the same time. Hence, the fault types of the inverters include single faults and composite faults.

These can be differentiated by two rules [31].

Rule 1. The maximum fault probability is greater than a boundary value $\varepsilon_{1}$, and the other fault probability value is less than a sure boundary value $\varepsilon_{2}$.

Rule 2. The difference value between the maximum fault probability and any other fault probability is greater than a sure boundary value $\varepsilon_{3}$.

Satisfying any one rule can be judged as a single fault. Other cases can be judged as composite failure. All probability results are expressed as percentages, and all faults are differentiated by rules. Using comprehensive maintenance records, test data, and expert knowledge considerations, set $\varepsilon_{1}=80 \%, \varepsilon_{2}=50 \%$ and $\varepsilon_{3}=20 \%$.

5.2. Case Inference. To verify the inference ability of the BIFIM of the inverter under incomplete evidence information, find out the causes of the fault and give a reasonable probability explanation; four kinds of incomplete evidence were reasoned and verified, and the results are summarized in Table 5. Cases 1-4 are provided for the fault inference under simple evidence with obvious support (Case 1), insufficient evidence information (Case 2), complex evidence without obvious support (Case 3), and evidence information conflict (Case 4), respectively. Case 2 only gives the information of two symptom nodes, which is a very incomplete symptom information illustrated in Figure 7, but the inference result is clear. Case 3 gives the information of occurrence of the four symptom nodes, which is more complete than case 2 , but the reasoning result is unclear and belongs to the case without obvious support. Case 4 presents multiple sources of evidence, both from the symptom layer 
TABLE 3: The CPT 3 of the BIFIM (the number of parent nodes is 3).

\begin{tabular}{lcccccrrrr}
\hline \multirow{2}{*}{ Node information } & \multirow{2}{*}{ Mark $\left(X_{i}\right)$} & \multicolumn{4}{c}{ Parents node state } \\
& & FFF & TFF & FTF & TTF & FFT & TFT & FTT & TTT \\
\hline DC-link failure & $X_{19}$ & 0.05 & 0.34 & 0.24 & 0.50 & 0.21 & 0.44 & 0.72 & 0.91 \\
Abnormal pulse voltage waveform & $X_{26}$ & 0.05 & 0.32 & 0.45 & 0.62 & 0.15 & 0.48 & 0.53 & 0.95 \\
\hline
\end{tabular}

TABLE 4: The CPT 4 of the BIFIM (the number of parent nodes is 4).

\begin{tabular}{lccccccc}
\hline & \multicolumn{3}{c}{ Node Information and Mark $\left(X_{i}\right)$} & & \multicolumn{3}{c}{ Node Information and Mark $\left(X_{i}\right)$} \\
Parents node state & A-phase failure & B-phase failure & C-phase failure & Parents node state & A-phase failure & B-phase failure & C-phase failure \\
& $X_{16}$ & $X_{17}$ & $X_{18}$ & & $X_{16}$ & $X_{17}$ \\
\hline FFFF & 0.01 & 0.01 & 0.01 & FFFT & 0.27 & 0.30 & 0.86 \\
TFFF & 0.34 & 0.22 & 0.34 & TFFT & 0.69 & 0.62 & 0.27 \\
FTFF & 0.23 & 0.33 & 0.38 & FTFT & 0.60 & 0.79 & 0.67 \\
TTFF & 0.72 & 0.58 & 0.80 & TTFT & 0.85 & 0.94 & 0.65 \\
FFTF & 0.34 & 0.36 & 0.38 & FFTT & 0.63 & 0.49 & 0.84 \\
TFTF & 0.41 & 0.49 & 0.55 & TFTT & 0.87 & 0.83 & 0.82 \\
FTTF & 0.77 & 0.61 & 0.56 & FTTT & 0.83 & 0.86 \\
TTTF & 0.94 & 0.95 & 0.86 & TTTT & 0.99 & 0.87 \\
\hline
\end{tabular}

and from the operation layer, which is also a possible situation in practice and more consistent with the results in practice.

The third column in Table 5 shows the evidence information given, the fourth column gives the probabilities of four faults in the middle layer, the fifth column presents the results of the fault types, the sixth column reveals the fault inference results, and the seventh column states the state combination probability values of the fault inference results.

To compare the influence of incomplete information on the results of fault inference, Case 3 in Table 5 is gradually put in to complete evidence to calculate the probability of the occurrence of each fault variable and analyze the changing trend of probability. The analysis process is summarized in Table 6 . The evidence numbered 8 is the complete symptom information collected when a certain inverter fault occurs. The probability value given in numbered 8 is that of the happiness of the fault layer variables under complete evidences. No. 1 evidence gives the evidence listed in Case 3 in Table 5. Evidences numbered 2-8 add gradually different numbers of evidence node information based on the No. 1 evidence. The single probability information of all the nodes in the fault layer is obtained under different degrees of evidence completeness, and the changing trend of probability is compared and analyzed.

5.3. Discussion. From the data in Table 5, the former two cases are belonging to single faults, while the latter two cases are composite faults.

Single Faults: Case 1 and Case 2. Case 1 provides incomplete evidence with obvious support: $X_{20}=\mathrm{T}, X_{21}=\mathrm{T}, X_{26}=\mathrm{T}$, and $X_{27}=\mathrm{F}$, which is illustrated in Figure 8(a), with green ovals representing the evidences of occurrence, orange ovals representing the evidences of nonoccurrence, blue ovals the for faults of occurrence, and gray ovals for the operation state likely appearing. $X_{16}$ appears to be supported by Case 1. The occurrence of $X_{16}$ is supported by $X_{20}, X_{21}$, and $X_{26}$. The occurrence of $X_{19}$ is supported by $X_{20}$ and $X_{21}$. The nonoccurrence of $X_{19}$ is supported by $X_{27}$. The occurrence of $X_{17}$ and $X_{18}$ is supported by $X_{26}$. From the evidence point of view, they obviously supported an A-phase fault $\left(X_{16}\right)$. The result of the BIFIM is as follows: $P\left(X_{16} \mid \psi\right)=82.73 \%$, and the rest of the probabilities are less than $\varepsilon_{2}$, which satisfies Rule 1 . The fault type of case 1 is a single fault. The result of the fault inference shows that the probability of the occurrence of A-phase failure $\left(X_{16}\right)$ is $82.73 \%$. The combination probability of various states shows that the probability of A-phase failure $\left(X_{16}\right)$ and the A-phase lower bridge arm pulse loss $\left(X_{4}\right)$ occurred at the same time is the maximum, and the first fault location is $X_{4}$.

Case 2 is demonstrated in Figure 8(b) under incomplete evidence information (serious incompleteness). There are only two evidences in this case: $X_{23}=\mathrm{T}$ and $X_{24}=\mathrm{T}$. The occurrence of $X_{17}$ and $X_{19}$ are supported by $X_{23}$. The occurrence of $X_{18}$ and $X_{19}$ is supported by $X_{24}$. From only the evidence point of view, $X_{17}, X_{18}$, and $X_{19}$ may occur, and it is difficult to judge the specific position of the fault. According to the judgement result of fault type by the BIFIM, which can be seen in the fourth column in Table 5, these four faults showed little differences in probability. However, $P\left(X_{19}\right.$ । $\psi)-P\left(X_{18} \mid \psi\right)=22.23 \%>20 \%$, which satisfies Rule 2. The fault type in this case is still a single fault. The result of the fault inference shows that the probability of the occurrence of a DC-link fault $\left(X_{19}\right)$ is $53.56 \%$. Although there are only two nodes of evidence information, it could still determine the location of the fault, which reflects the strong inference ability under incomplete evidence information. The combination probability of various states shows that the probability of both the DC-link fault $\left(X_{19}\right)$ and capacitance parameter weakness $\left(X_{15}\right)$ occurred at the same time as the maximum, and the first fault location is $X_{15}$. 


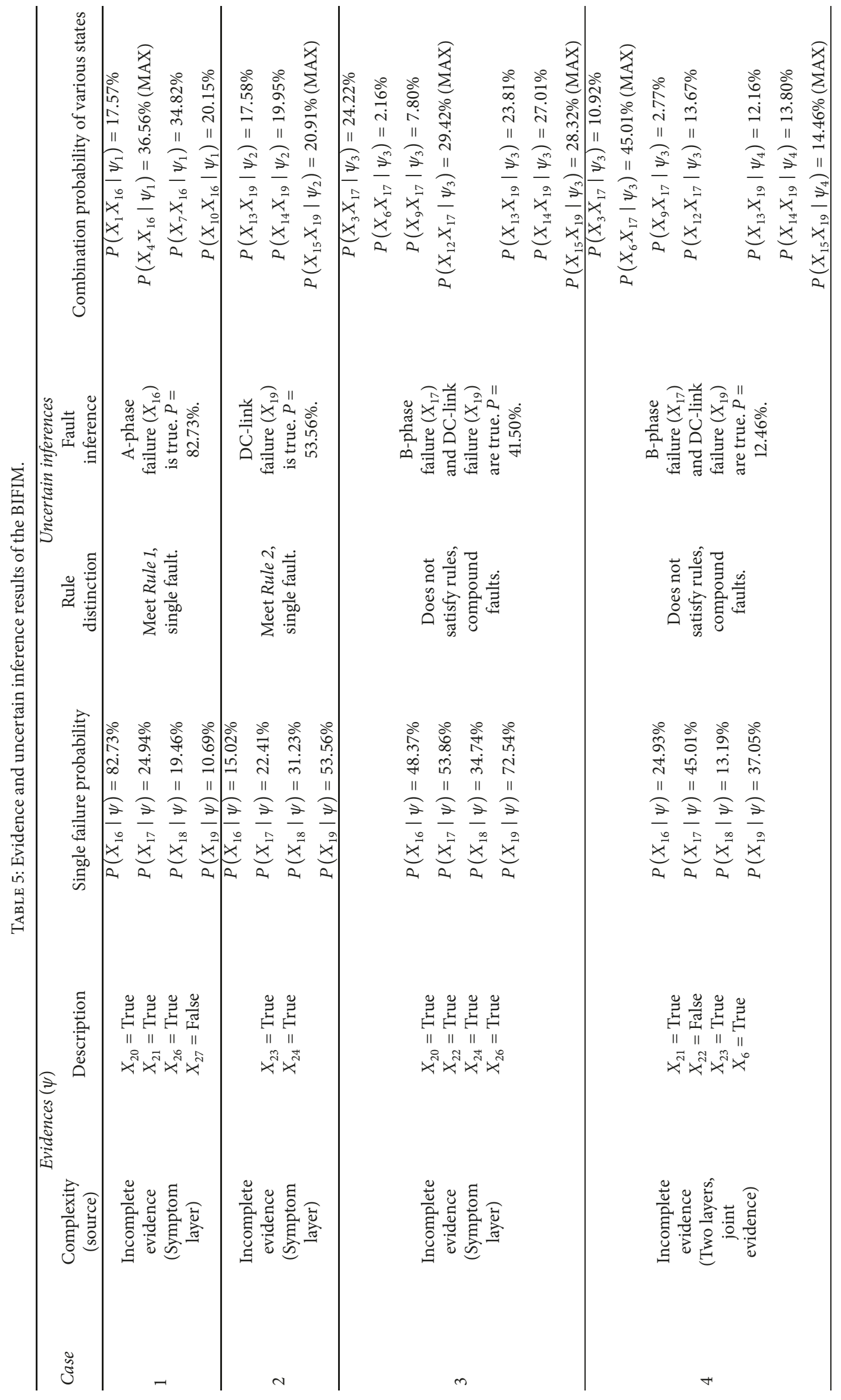


TABLE 6: Comparison of results under different degrees of completeness of evidence.

\begin{tabular}{|c|c|c|c|c|c|c|c|c|c|c|c|c|c|c|c|}
\hline \multirow{2}{*}{ Num. } & \multicolumn{11}{|c|}{ Node status } & \multicolumn{4}{|c|}{ Result probability comparison } \\
\hline & $X_{20}$ & $X_{21}$ & $X_{22}$ & $X_{23}$ & $X_{24}$ & $X_{25}$ & $X_{26}$ & $X_{27}$ & $X_{28}$ & $X_{29}$ & $X_{13}$ & $X_{16}$ & $X_{17}$ & $X_{18}$ & $X_{19}$ \\
\hline 1 & $\mathrm{~T}$ & & $\mathrm{~T}$ & & $\mathrm{~T}$ & & $\mathrm{~T}$ & & & & & 0.4837 & 0.5386 & 0.3474 & 0.7254 \\
\hline 2 & $\mathrm{~T}$ & & $\mathrm{~T}$ & $\mathrm{~T}$ & $\mathrm{~T}$ & & $\mathrm{~T}$ & & & & & 0.4122 & 0.7273 & 0.3055 & 0.8108 \\
\hline 3 & $\mathrm{~T}$ & $\mathrm{~F}$ & $\mathrm{~T}$ & $\mathrm{~T}$ & $\mathrm{~T}$ & & $\mathrm{~T}$ & & & & & 0.3269 & 0.7444 & 0.3430 & 0.6857 \\
\hline 4 & $\mathrm{~T}$ & $\mathrm{~F}$ & $\mathrm{~T}$ & $\mathrm{~T}$ & $\mathrm{~T}$ & & $\mathrm{~T}$ & & $\mathrm{~T}$ & & & 0.2366 & 0.7734 & 0.2620 & 0.9441 \\
\hline 5 & $\mathrm{~T}$ & $\mathrm{~F}$ & $\mathrm{~T}$ & $\mathrm{~T}$ & $\mathrm{~T}$ & & $\mathrm{~T}$ & $\mathrm{~F}$ & $\mathrm{~T}$ & & & 0.3436 & 0.7390 & 0.3580 & 0.6387 \\
\hline 6 & $\mathrm{~T}$ & $\mathrm{~F}$ & $\mathrm{~T}$ & $\mathrm{~T}$ & $\mathrm{~T}$ & & $\mathrm{~T}$ & $\mathrm{~F}$ & $\mathrm{~T}$ & & $\mathrm{~T}$ & 0.2656 & 0.7641 & 0.2881 & 0.8609 \\
\hline 7 & $\mathrm{~T}$ & F & $\mathrm{T}$ & $\mathrm{T}$ & $\mathrm{T}$ & & $\mathrm{T}$ & F & $\mathrm{T}$ & $\mathrm{T}$ & & 0.2282 & 0.7761 & 0.2545 & 0.9680 \\
\hline 8 & $\mathrm{~T}$ & $\mathrm{~F}$ & $\mathrm{~T}$ & $\mathrm{~T}$ & $\mathrm{~T}$ & $\mathrm{~F}$ & $\mathrm{~T}$ & $\mathrm{~F}$ & $\mathrm{~T}$ & $\mathrm{~T}$ & & 0.2306 & 0.7912 & 0.1149 & 0.9563 \\
\hline
\end{tabular}

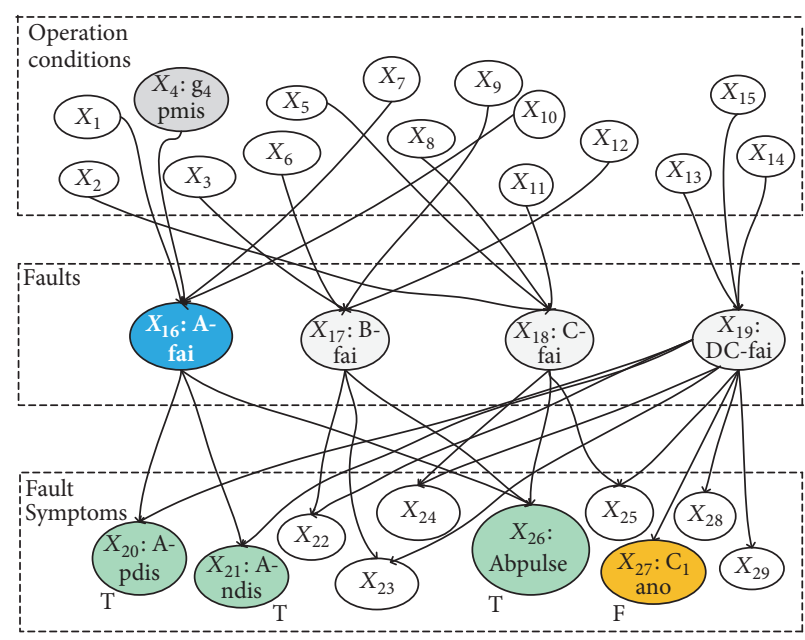

(a) Case 1

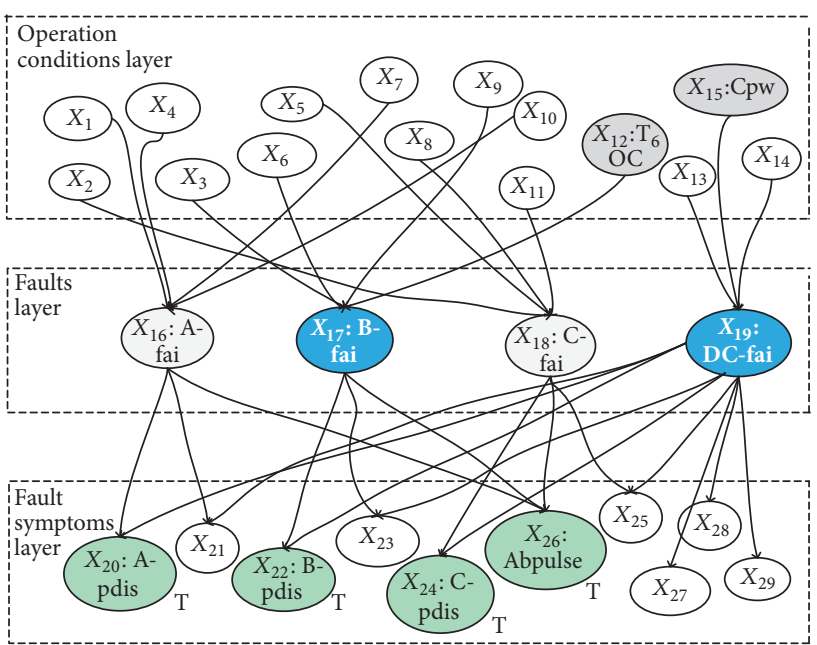

(c) Case 3

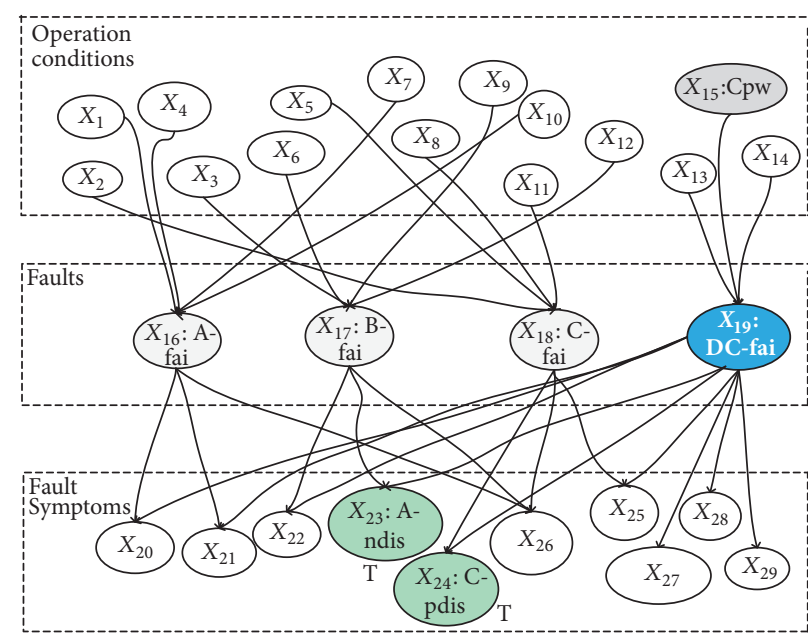

(b) Case 2

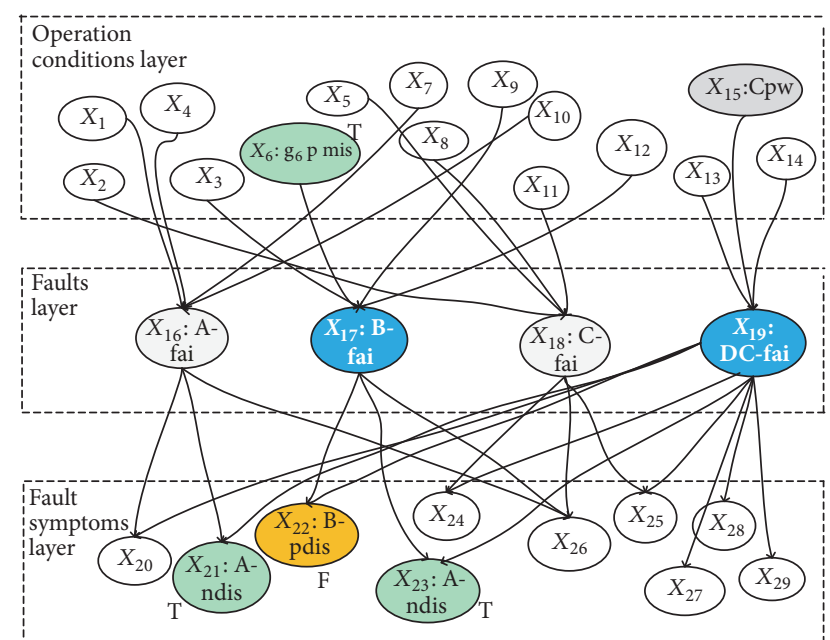

(d) Case 4

FIGURE 8: Inference results from different evidence.

Composite Faults: Case 3 and Case 4. Case 3 provides complex evidence without evident support: $X_{20}=\mathrm{T}, X_{22}=\mathrm{T}, X_{24}=\mathrm{T}$, and $X_{26}=$ T shown in Figure $8(\mathrm{c})$. The occurrence of $X_{16}, X_{17}$, and $X_{18}$ are supported by $X_{20}, X_{22}$, and $X_{24}$, respectively. The simultaneous occurrence of $X_{16}, X_{17}$, and $X_{18}$ are supported by $X_{26}$. The occurrence of $X_{19}$ is supported by $X_{20}, X_{22}$, and $X_{24}$. From the evidence information given, $X_{16}, X_{17}$, and $X_{18}$ all have one supporting evidence information which does not obviously support any single fault and could not judge whether the three faults occur. $X_{19}$ has three supporting evidences, so $X_{19}$ have a higher probability of occurrence than that of others. The calculation results of a single fault 
based on the BIFIM shows that the A-phase fault $\left(X_{16}\right)$, Bphase fault $\left(X_{17}\right)$, and DC-link fault $\left(X_{19}\right)$ may occur in the 4 th column in Table 5 . The result of the rule differentiation shows that the case does not satisfy any one rule and the fault type is a composite fault. The results of the fault inference and the combination probability of various states show that there are likely B-phase faults $\left(X_{17}\right)$ and DC-link faults $\left(X_{19}\right)$ at the same time, which are caused by the B-phase lower bridge arm IGBT OC $\left(X_{12}\right)$ and the capacitance parameter variation $\left(X_{15}\right)$, respectively. As the fault type is a compound fault, two failure points are located first: $X_{12}$ and $X_{15}$, respectively.

Case 4 provides joint evidence with confliction of the evidential information. Evidence comes from the fault symptom layer and the operation condition layer. $X_{21}=\mathrm{T}, X_{22}=\mathrm{F}, X_{23}$ $=\mathrm{T}$, and $X_{6}=\mathrm{T}$ shown in Figure $8(\mathrm{~d})$. The nonoccurrence of $X_{17}$ and $X_{19}$ were supported by $X_{22}$, while the occurrence of $X_{17}$ and $X_{19}$ is supported by $X_{23}$. This is the conflict among the evidence information in the symptom layer. The nonoccurrence of $X_{17}$ is supported by $X_{22}$ and the occurrence of $X_{17}$ is supported by $X_{6}$, which shows that there are both evidence information conflict and the different layer conflict between symptom layer and condition layer. From the evidence given, if $X_{21}$ and $X_{23}$ occur, then $X_{16}, X_{17}$, and $X_{19}$ may occur, and the probability of $X_{19}$ occurring is higher. As $X_{22}$ does not occur, the probability of the occurrence of $X_{17}$ and $X_{19}$ decreases and the probability of the happiness of $X_{17}$ rises due to the occurrence of the operational condition layer evidence $X_{6}$. The result of the rule differentiation shows that this case does not satisfy any one rule and the fault type is a composite fault. The probabilistic results of the fault inference and state combination show that there are probably B-phase faults $\left(X_{17}\right)$ and DC-link faults $\left(X_{19}\right)$ at the same time, which are caused by the B-phase lower bridge arm pulse loss $\left(X_{6}\right.$, has occurred $)$ and the capacitance parameter variation $\left(X_{15}\right)$, respectively. As the fault type is a composite fault, two failure points to be located first are $X_{6}$ and $X_{15}$, respectively.

From the evidence and inference results in Table 5, we can see the following.

When $X_{20}, X_{22}, X_{24}$, and $X_{26}$ occur, as $X_{20}, X_{22}$, and $X_{24}$ all support the occurrence of $X_{19}$, the probability of the occurrence of $X_{19}$ is higher relative to $X_{16}, X_{17}$, and $X_{18}$, but the rule is not enough to determine the single fault. In the case of the evidence information from numbered 3 (Case 3 ), it is still difficult to distinguish the probability of fault occurrence for $X_{16}, X_{17}$, and $X_{18}$. In this respect, it is better to obtain other evidences to make the probability of the fault more distinguishable to be able to locate the fault accurately.

Incomplete Information Inference. The following evidence is added continuously for further analysis.

By adding evidence information $X_{23}=$ T, Figures 7 and 8 show that $X_{23}$ is the symptom information of $X_{17}$ and $X_{19}$, so the probability of the occurrence of $X_{17}$ and $X_{19}$ increase to 0.7273 and 0.8108 , respectively, which is demonstrated in Table 6. By adding evidence information $X_{21}=\mathrm{F}$, where $X_{21}$ is the symptom information of $X_{19}$, the probability of the occurrence of $X_{19}$ reduces to 0.6857 . It can be concluded that the more symptoms determining the occurrence of a fault happen, the greater the probability of the occurrence of the fault is.

Adding evidence information $X_{28}=\mathrm{T}, X_{28}$ is only the symptom information of $X_{19}$; that is, there is only one parent node. It is found that the probability of the occurrence of $X_{19}$ increases significantly to 0.9441 . Adding evidence $X_{27}=\mathrm{F}$, which is only the symptom information of $X_{19}$, it is found that the probability of the occurrence of $X_{19}$ significantly reduces to 0.6387 . When the evidence information provided by conflict existing in the symptom layer, it is difficult to distinguish the probability of failure. In this way, it is better to obtain the extra evidence information of the state layer to further determine the fault. Adding evidence information $X_{13}=\mathrm{T}$ numbered 6 in Table 6 , the probability of the occurrence of $X_{19}$ increases significantly to $0.8609 . X_{13}$ is the variable in the operation condition layer. Combined with the analysis results in row 5 (numbered 5), the evidence information of variables of the operation conditions layer has a great impact on the probability of the occurrence of supporting fault variables even though the symptom information conflicts.

Adding evidence information $X_{29}=\mathrm{T}$, which is only the symptom information of $X_{19}$, we find that the probability of the occurrence of $X_{19}$ increases dramatically to 0.9680 . From the changing trend of $X_{19}$ after adding the evidence $X_{28}, X_{27}$, and $X_{29}$, it can be seen when the unique evidence (supporting only one fault variable) appears, whether it occurred or not, it has a great influence on the probability of the occurrence of supporting fault variables. By adding evidence information $X_{25}=\mathrm{F}$, the fault probability under the complete evidence information could be directly located from the results.

From the overall trend of the fault variables numbered 1 to 8 , with the addition of evidence information, the single probability of the fault layer nodes changes constantly, as illustrated in Figure 9. With the addition of evidence, the probability value tends to approach the probability value in the case of complete evidence. Under the complete evidence information numbered 8 , it seems that the probability of $X_{19}(0.9563>80 \%)$ is certain to occur according to Rule 1. However, the probabilities of $X_{17}(0.7912 \approx 80 \%)$ and $X_{19}$ $(0.9563>80 \%)$ confuse the occurrence of the fault nodes. Namely, even though the complete information is acquired and, it is a challenging task to inference which fault is sure to occur. It is concluded that a composite fault, Bphase faults $\left(X_{17}\right)$, and DC-link faults $\left(X_{19}\right)$ is likely to occur as described in Case 3 (Figure 8). For the incomplete information numbered 1 to 7 , the other 6 numbers 2-7 except for number 1 have the similar inference results as number 8 (shown in the light blue area). The evidence numbered 1 neither satisfies Rule 1 nor Rule 2 and the distinction among the four faults is too obscure (shown in the light blue area). As can be seen from Figure 9, the results of inferences with the other evidence numbered 2 to 7 are that $X_{17}$ and $X_{19}$ occur simultaneously, whose probability is obviously much higher than the remaining two faults and the corresponding curves of $X_{17}$ and $X_{19}$ deviate far from ones of $X_{16}$ and $X_{18}$. 


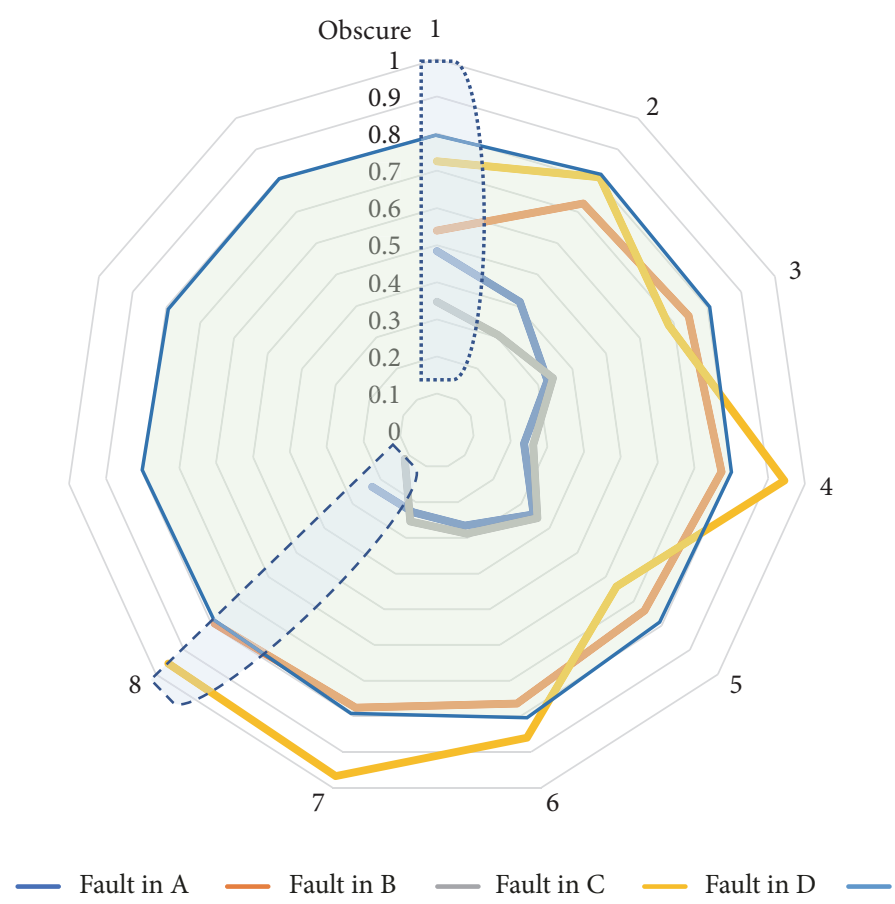

FIGURE 9: Inference based on the degree of evidence incompleteness.

\section{Conclusions}

In the study, an intelligent fault inference model based on a three-layer BN called BIFIM is proposed to improve the inference and diagnostic ability of the inverter's faults in the case of incomplete information. The Bayesian network consists of the inverter's operation conditions, inverter's faults, and fault symptoms combined with the knowledge of field experts and the experiences of field technicians as well as the historical data of the inverter's operation. The main conclusions are as follows.

(1) The operation state of the inverter is the first layer of the BIFIM. Compared with the general two layer and variable independent naive Bayesian networks, the inference network can infer a variety of complex faults. The inference network is more consistent with experts' inference thinking and inference strategies. Embodying stronger intelligence, it could deal with a variety of complex causation and uncertain events.

(2) Through the analysis of many types of evidence, the inference network could deduce single faults under complete evidence, single faults under incomplete information, and complex faults under incomplete information. For some incomplete evidence information, the same reasoning result can be given under the condition of complete information, and, in most cases, the reasoning result is similar to that under the condition of complete information. Thus, BIFIM shows its strong inference ability under incomplete information.

\section{Data Availability}

The data used to support the findings of this study are available from the corresponding author upon request.

\section{Conflicts of Interest}

The authors declare no conflicts of interest.

\section{Authors' Contributions}

All authors were involved in the whole research design and analysis, writing, and verification. All authors approved the final version of the manuscript.

\section{Acknowledgments}

Thank are due to Zhao gu II Mine for providing data support for this research. This research was funded by the National Key Research and Development Program, Grant no. 2016 YFC0600906.

\section{References}

[1] S. Li, C. Xia, and X. Zhou, "Disturbance rejection control method for permanent magnet synchronous motor speedregulation system," Mechatronics, vol. 22, no. 6, pp. 706-714, 2012.

[2] L. H. Zhang, Y. W. Hu, and W. X. Huang, "Fault diagnosis and tolerant techniques of inverter in three-phase variable frequency drive system," Transactions of China Electrotechnical Society, vol. 19, pp. 1-9, 2004.

[3] B. Tabbache, M. Benbouzid, A. Kheloui, J.-M. Bourgeot, and A. Mamoune, "An improved fault-tolerant control scheme for PWM inverter-fed induction motor-based EVs," ISA Transactions, vol. 52, no. 6, pp. 862-869, 2013.

[4] G. Tang, B. Pang, T. Tian, and C. Zhou, "Fault diagnosis of rolling bearings based on improved fast spectral correlation and 
optimized random forest," Applied Sciences, vol. 8, no. 10, p. 1859, 2018.

[5] N. P. Nguyen and S. K. Hong, "Sliding mode Thau observer for actuator fault diagnosis of quadcopter UAVs," Applied Sciences, vol. 8, no. 10, p. 1893, 2018.

[6] Y. Peng, X. Wang, L. Guo, Y. Wang, and Q. Deng, "An efficient network coding-based fault-tolerant mechanism in wban for smart healthcare monitoring systems," Applied Sciences, vol. 7, no. 8, p. 817, 2017.

[7] X. Wang and Y. Shen, "Fault-tolerant control strategy of a wind energy conversion system considering multiple fault reconstruction," Applied Sciences, vol. 8, no. 5, p. 794, 2018.

[8] B. G. Xu, "Intelligent fault inference for rotating flexible rotors using Bayesian belief network," Expert Systems with Applications, vol. 39, no. 1, pp. 816-822, 2012.

[9] G. Krishnamoorthy, P. Ashok, and D. Tesar, "Simultaneous sensor and process fault detection and isolation in multipleinput-multiple-output systems," IEEE Systems Journal, vol. 9, no. 2, pp. 335-349, 2015.

[10] X. Guo, C. Shen, and L. Chen, "Deep fault recognizer: an integrated model to denoise and extract features for fault diagnosis in rotating machinery," Applied Sciences, vol. 7, no. 1, p. 41, 2017.

[11] M.-H. Wang, Y.-F. Tseng, H.-C. Chen, and K.-H. Chao, "A novel clustering algorithm based on the extension theory and genetic algorithm," Expert Systems with Applications, vol. 36, no. 4, pp. 8269-8276, 2009.

[12] M. A. Mohammed, M. K. A. Ghani, N. Arunkumar et al., "Genetic case-based reasoning for improved mobile phone faults diagnosis," Computers Electrical Engineering, vol. 71, pp. 212-222, 2018.

[13] G. Mani and J. Jerome, "Intuitionistic fuzzy expert system based fault diagnosis using dissolved gas analysis for power transformer," Journal of Electrical Engineering \& Technology, vol. 9, no. 6, pp. 2058-2064, 2014.

[14] J.-D. Wu and C.-H. Liu, "An expert system for fault diagnosis in internal combustion engines using wavelet packet transform and neural network," Expert Systems with Applications, vol. 36, no. 3, pp. 4278-4286, 2009.

[15] N. Saravanan, S. Cholairajan, and K. I. Ramachandran, "Vibration-based fault diagnosis of spur bevel gear box using fuzzy technique," Expert Systems with Applications, vol. 36, no. 2, pp. 3119-3135, 2009.

[16] S. X. Ni, Y. F. Zhang, H. Yi, and X. F. Liang, "Intelligent fault diagnosis method based on fault tree," Journal of Shanghai Jiaotong University, vol. 8, pp. 1372-1375, 2008.

[17] A. Nouri.Gharahasanlou, A. Mokhtarei, A. Khodayarei, and M. Ataei, "Fault tree analysis of failure cause of crushing plant and mixing bed hall at Khoy cement factory in Iran," Case Studies in Engineering Failure Analysis, vol. 2, no. 1, pp. 33-38, 2014.

[18] B.-G. Park, K.-J. Lee, R.-Y. Kim, T.-S. Kim, J.-S. Ryu, and D.-S. Hyun, "Simple fault diagnosis based on operating characteristic of brushless direct-current motor drives," IEEE Transactions on Industrial Electronics, vol. 58, no. 5, pp. 1586-1593, 2011.

[19] S. Ouni, J. Rodriguez, M. Shahbazi et al., "A fast and simple method to detect short circuit fault in cascaded H-bridge multilevel inverter," in Proceedings of the 2015 IEEE International Conference on Industrial Technology (ICIT), pp. 866-871, Seville, Spain, March 2015.

[20] Z. Liu, Y. Liu, H. Shan, B. Cai, Q. Huang, and Y. Deng, "A fault diagnosis methodology for gear pump based on eemd and bayesian network," PLoS ONE, vol. 10, no. 5, p. e0125703, 2015.
[21] J. Liu, B. Ma, and Z. Jiang, "A study of probabilistic diagnosis method for three kinds of internal combustion engine faults based on the graphical model," Mathematical Problems in Engineering, vol. 2019, Article ID 8156450, 14 pages, 2019.

[22] Y. Luo, K. Li, Y. Li, D. Cai, C. Zhao, and Q. Meng, “Three-layer bayesian network for classification of complex power quality disturbances," IEEE Transactions on Industrial Informatics, vol. 14, no. 9, pp. 3997-4006, 2018.

[23] O. J. Mengshoel, M. Chavira, K. Cascio, S. Poll, A. Darwiche, and S. Uckun, "Probabilistic model-based diagnosis: An electrical power system case study," IEEE Transactions on Systems, Man, and Cybernetics: Systems, vol. 40, no. 5, pp. 874-885, 2010.

[24] L. W. Zhang and H. P. Guo, Introduction to Bayesian Networks, Science Press, Beijing, China, 2006.

[25] J. C. Ramírez, G. Muñoz, and L. Gutierrez, "Fault diagnosis in an industrial process using Bayesian Networks: Application of the junction tree algorithm," in Proceedings of the CERMA 2009 - Electronics Robotics and Automotive Mechanics Conference, pp. 301-306, Mexico, September 2009.

[26] F. V. Jensen and T. D. Nielsen, Bayesian Networks and Decision Graphs, Springer Science \& Business Media, New York, NY, USA, 2007.

[27] H. Y. Yan, Research on exact inference algorithm in Bayesian networks [Master, thesis], Jilin University, Jilin, China, 2006.

[28] D. G. Xu, X. F. Liu, and Y. Yu, "A survey on fault diagnosis and tolerant control of inverters," Transactions of China Electrotechnical Society, vol. 30, no. 21, pp. 1-12, 2015.

[29] T. Wang, J. Qi, H. Xu, Y. Wang, L. Liu, and D. Gao, "Fault diagnosis method based on fft-rpca-svm for cascaded-multilevel inverter," ISA Transactions, vol. 60, pp. 156-163, 2016.

[30] S. Hameg, M. Lazri, and S. Ameur, "Using naive Bayes classifier for classification of convective rainfall intensities based on spectral characteristics retrieved from SEVIRI," Journal of Earth System Science, vol. 125, no. 5, pp. 945-955, 2016.

[31] Y. Zhao, F. Xiao, and S. W. Wang, "An intelligent chiller fault detection and diagnosis methodology using Bayesian belief network," Energy \& Buildings, vol. 57, pp. 278-288, 2013. 


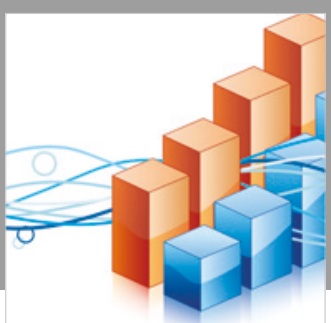

Advances in

Operations Research

\section{-n-m}
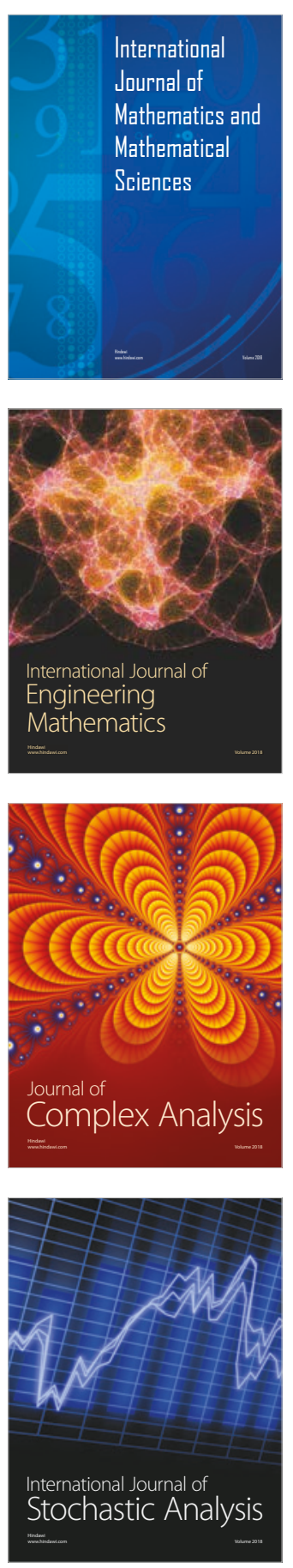
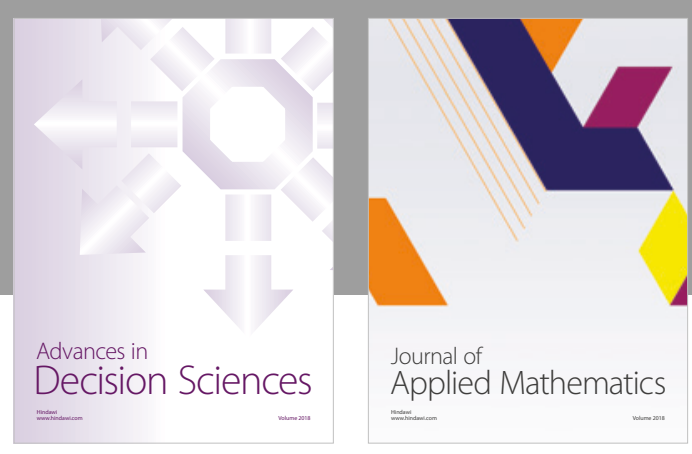

Journal of

Applied Mathematics
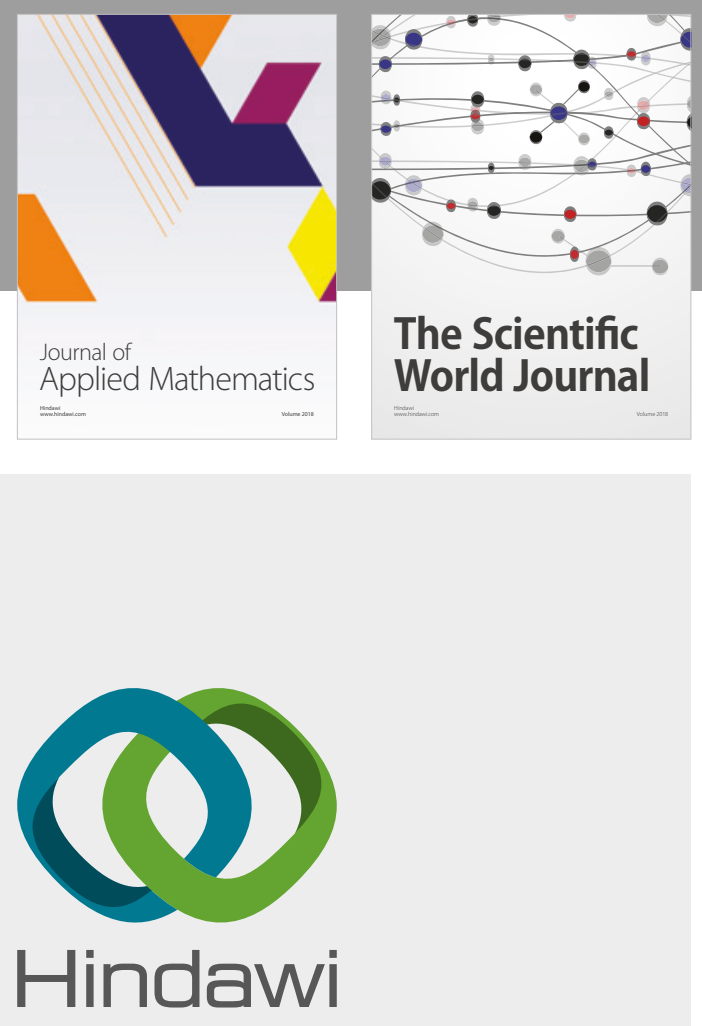

Submit your manuscripts at

www.hindawi.com

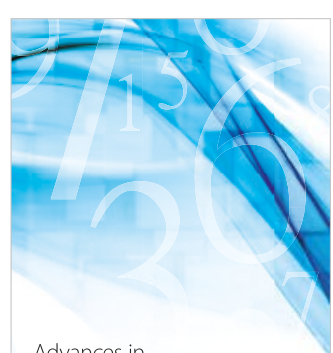

Advances in
Numerical Analysis
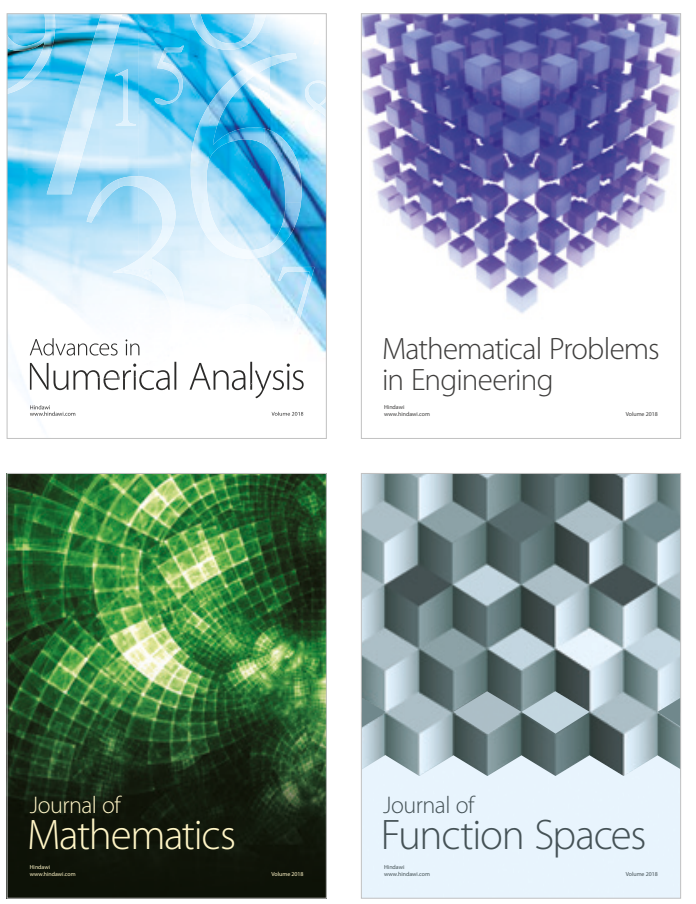

Mathematical Problems in Engineering

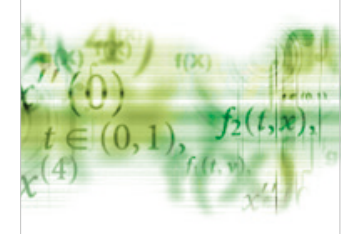

International Journal of

Differential Equations

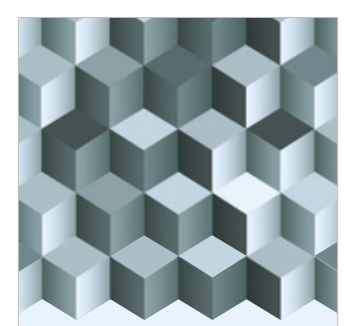

Journal of

Function Spaces

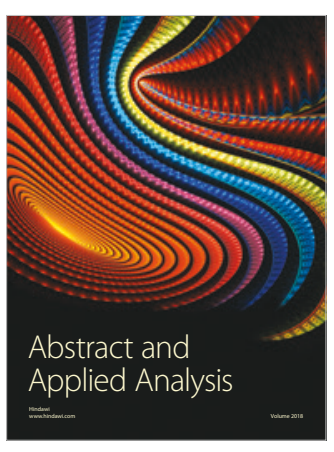

The Scientific

World Journal

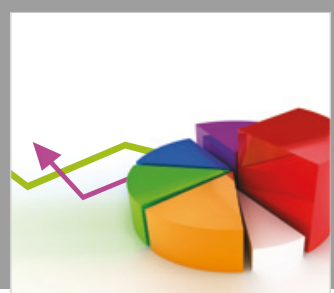

Journal of

Probability and Statistics
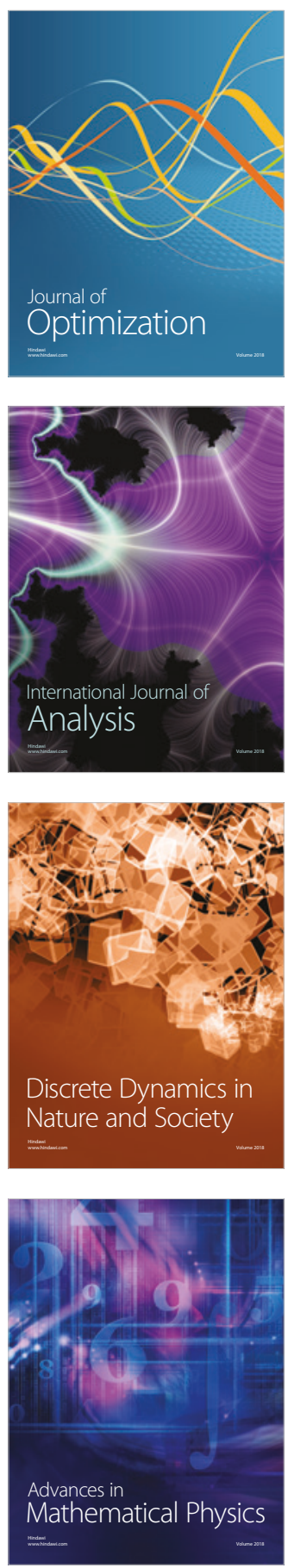